\title{
A simple technique for a.c. conductivity measurements
}

\author{
R PADMA SUVARNA, K RAGHAVENDRA RAO* and K SUBBARANGAIAH \\ Department of Physics and Electronics, Sri Krishnadevaraya University, Anantapur 515 003, India
}

MS received 14 May 2001; revised 23 September 2002

\begin{abstract}
An inexpensive, indigenous and a simple electronic instrument based on voltage follower, currentto-voltage converter, zero crossing detector and a phase detector has been developed for measurement of a.c. conductivity. Real and imaginary parts of complex impedance are determined for a given sample as a function of frequency and the given sample is represented by a pure electronic model.
\end{abstract}

Keywords. A.c. conductivity; complex impedance spectroscopy; bulk resistance; grain boundaries.

\section{Introduction}

A.c. conductivity is one of the studies done on solids in order to characterize the bulk resistance of the crystalline sample. Measurement of a.c. conductivity can be done by different techniques. The currently used technique is the complex impedance spectroscopy. This study also gives information on electrical properties of materials and their interface with electronically conducting electrodes. The complex impedance spectroscopy measurement of a.c. conductivity is based on studies made on the measurement of cell impedance/admittance over a range of temperatures and frequencies and analysing them in complex impedance plane (Bauerle 1969; Macdonald 1987). This is particularly characterized by the measurement and analysis of $Z$ (impedance), $Y$ (admittance) and plotting of these functions in the complex plane which is known as Nyquist diagrams.

Impedance is a more general concept than resistance because it takes phase differences into account. In a.c., the resistance, $R$, is replaced by the impedance, $Z$, which is the sum of resistance and reactance. Impedance can be written as

$$
Z=Z^{\prime}+Z^{\prime \prime}
$$

where $Z^{\prime}$ is the real part and $Z^{\prime \prime}$ the imaginary part of $Z$. $Z$ is a vector quantity and may be plotted in the plane with either rectangular or polar coordinates as shown in figure 1.

The two rectangular coordinate values are

$$
\begin{aligned}
& \operatorname{Re}(Z)=Z^{\prime}=|Z| \cos \phi, \\
& \operatorname{Im}(Z)=Z^{\prime \prime}=|Z| \sin \phi,
\end{aligned}
$$

with phase angle $\phi=\tan ^{-1}\left(Z^{\prime} / Z^{\prime \prime}\right)$ and $|Z|=\left[\left(Z^{\prime}\right)^{2}+\left(Z^{\prime \prime}\right)^{2}\right]^{1 / 2}$.

\footnotetext{
*Author for correspondence
}

In polar form, $Z$ may be written as

$$
Z(\omega)=|Z| \exp (j \omega)
$$

where

$$
\exp (j \omega)=\cos (\phi)+j \sin (\phi) .
$$

In general, $Z$ is frequency dependant. Impedance spectroscopy consists of the measurement of $Z(\omega)$ over a wide frequency range. It is from the resulting structure of $Z(\omega)$ vs $\omega$ one derives information about the electrical properties of the electrode material system. Impedance plane plots for series and parallel combinations of $R$ and $C$ are shown in figure 2 .

A symmetrical solid cell system can be represented by the simplified general equivalent circuit as shown in figure 3 . $Z_{\mathrm{e}}$ is the bulk electrolyte impedance contribution, $Z_{\mathrm{i}}$ the sum of both interface impedance contributions (on both sides of the electrolyte) and $C_{\mathrm{g}}$ the geometrical cell capacity. The use of admittance plane plotting (ColeCole plot) for accurate conductivity determination of solid electrolytes was introduced by Bauerle (1969). An automatic system for frequency dependant impedance measurement based on computer controlled network which can measure up to $175^{\circ} \mathrm{C}$ was developed by Staudt (1981) and Schon (1989). A microprocessor based Hewlett Packard 4192A low frequency impedance analyser was developed by Boukamp (1984), which overcomes the difficulty of measuring impedance in a specific range of frequency. The a.c. conductivity of solid electrolyte is analysed by Bruce and West (1983) in terms of equivalent circuit consisting of resistors and capacitors. They conducted two terminal a.c. measurements over the frequency range $10^{-3}-10^{7} \mathrm{~Hz}$ using a combination of bridge and automated phase sensitive detection techniques. Balaya and Sunandana (1989) designed an electronic system based on quadrature oscillator, current-to-voltage converter and phase sensitive detector for measurement of a.c. conductivity and complex impedance over a range 
of frequencies up to $60 \mathrm{kHz}$. It is evident from the review of literature that though bulk of the effort has gone into conductivity measurements by various techniques, very little work has been reported on the instrumentation aspects of the developed techniques. All the measuring techniques seem to be quite expensive and complex. To represent the given sample by pure electronic model calls for the use of complex impedance technique. The impedance spectrum is essential to obtain wealth of information about the sample. This spectrum can be obtained through several electronic techniques. From the above view points, it is clear that the complex impedance spectroscopic measurement of a.c. conductivity is a widely used method. Hence, a modest attempt is made here to develop an inexpensive, indigenous and simple electronic instrument for the measurement of real and imaginary parts of complex impedance of the given sample.

\section{Experimental}

The block diagram of the circuit is shown in figure 4 . It essentially consists of signal generator, voltage follower,

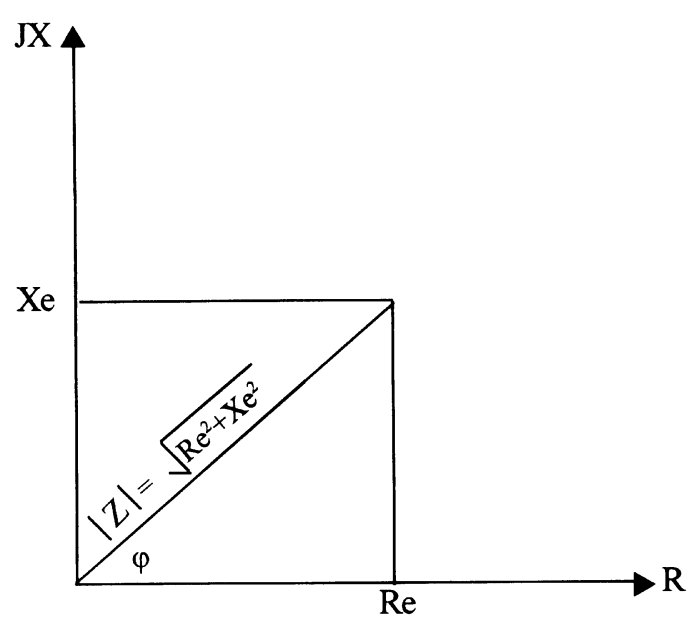

Figure 1. Impedance vector representation.
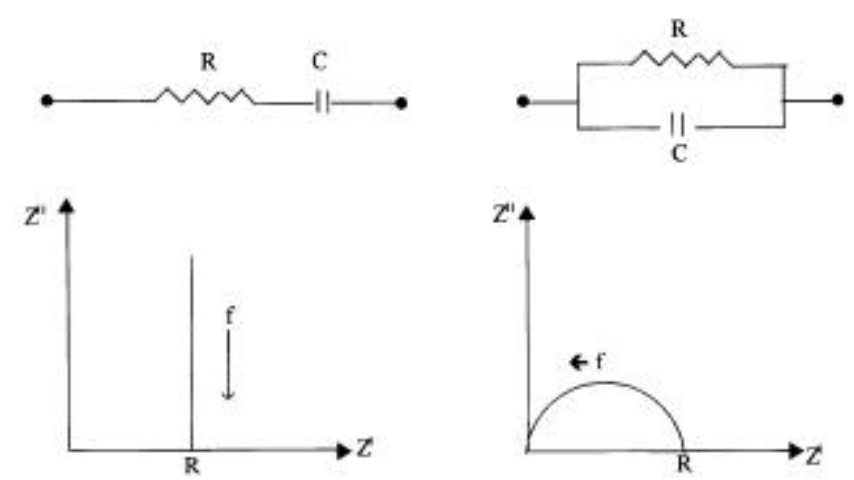

Figure 2. Impedance plots for series and parallel combinations of $R$ and $C$. current-to-voltage converter, zero crossing detector and phase detector.

Signal generator: Scientific (HM5030-4) make function generator was used as the signal generator in the present work. This function generator has provision for sine, square and triangular wave of both analog and TTL compatible outputs.

Voltage follower: The voltage follower was constructed using low offset, low drift, JFET input operational amplifier LF411 (National Semiconductors 1998). The reason for using such an Op. Amp. is that it has internally trimmed offset voltage, high input impedance, high slew rate, wide band width and fast settling time. Thus, it works as a buffer between the signal generator and other circuits, such as, the experimental cell and zero crossing detector.

Current-to-voltage converter $(C V C)$ : The current passing through the sample placed in the experimental cell has to be detected and measured precisely. This is accomplished with a low noise precision difet Op. Amp. OPA111 (Burr-Brown 1998). It has desirable features such as low noise, low bias current, low offset and low drift. Verylow bias current is obtained by dielectric isolation with on-chip guarding. This design facilitates the precise measurement of current resulting from the sample. The CVC designed using OPA111 is shown in figure 5. This Op. Amp. provides an input impedance of $10^{14} \mathrm{ohms}$ with low bias current of $\pm 1 \mathrm{pA}$. This industrially standard

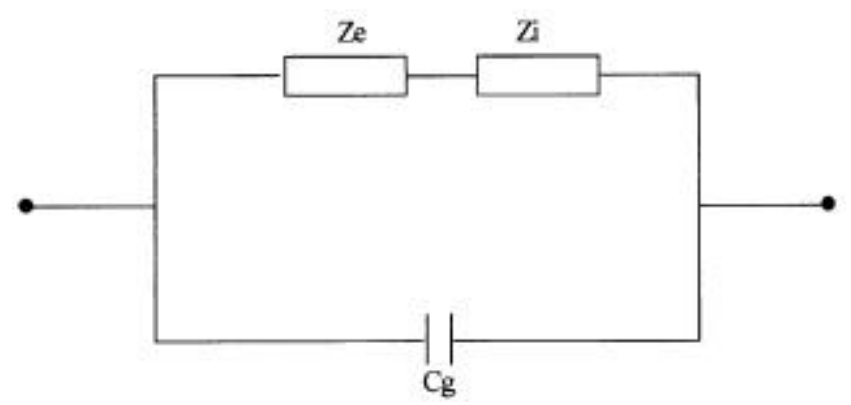

Figure 3. Equivalent circuit of a symmetrical solid cell.

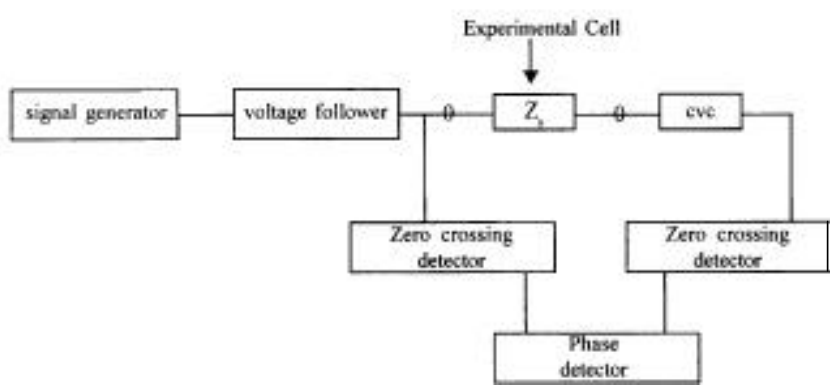

Figure 4. Block diagram of experimental setup. 
Op. Amp. in conjunction with the modified cell design yielded good results as compared to the previous design by Balaya and Sunandana (1989). This operates on the principle that current through the input impedance $\left(Z_{\mathrm{s}}\right)$ and the feed back resistor $\left(R_{\mathrm{f}}\right)$ will always be equal in order to maintain zero voltage difference at the inverting terminal. If $Z_{\mathrm{s}}$ is purely resistive, there will be change in the amplitude. But if $Z_{\mathrm{s}}$ is capacitive/inductive then there will be a phase difference between output and input signals and also a change in their amplitudes. Generally, if $Z_{\mathrm{s}}$ is a complex impedance which involves both resistive and capacitive/inductive reactances, the output signal undergoes both amplitude and phase modulations. Non-

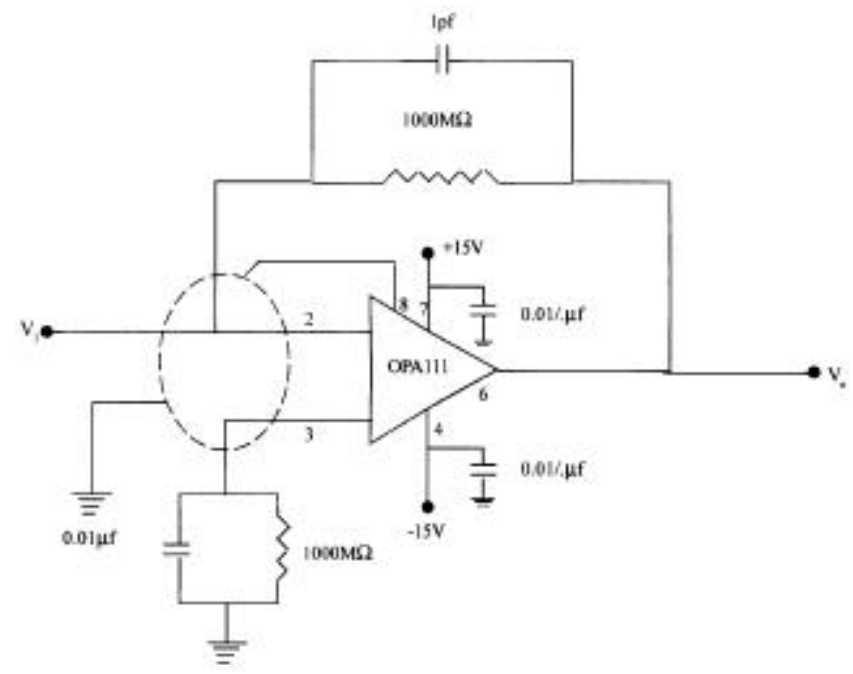

Figure 5. Current-to-voltage convertor.

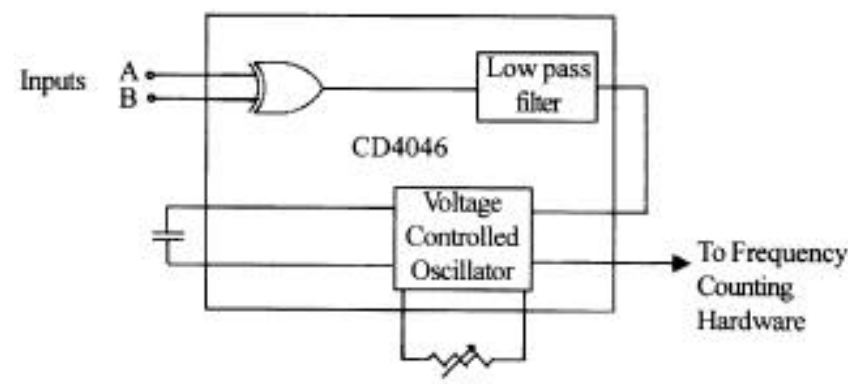

Figure 6. Phase detection using PLL. ranging from $1 \mathrm{k} \Omega$ to $1000 \mathrm{M} \Omega$ were ıck loop.

tector: The zero crossing detectors using the Op. Amp. LM 301. The Op. out the internal frequency compensation so it slews more rapidly than the Op. utputs of the ZCDs are connected to the

The measurement of phase difference als of same frequency is carried out by like diode phase detector, double balanrr etc. The new method adopted in this on of phase difference into proportional proportional frequency using PLL IC agram is shown in figure 6.

zd loop consists of phase detector (XOR), Id voltage controlled oscillator (VCO). $\mathrm{R}$ gate are fed from the outputs of comces an output voltage which has a d.c. rtional to the phase difference between rals. The VCO converts the d.c. voltage Iter into proportional frequency. As the ut signal changes, a change in phase tput signals of the comparator (inputs to :e a change in d.c. voltage in such a way 'quency of VCO. This feature (convert) proportional frequency within a single ; exploited in accomplishing the task of nt. This type of phase measurement is sal and compact as compared to the f Balaya and Sunandana (1989).

Ifiguration placed in an electrically vity cell is connected to the inverting the sinusoidal signal, $V_{1} \sin (\omega t)$ from zenerator is allowed to pass through the it voltage becomes $V_{0} \sin (\omega t+\phi)$, where troduced by the sample. By measuring voltages of the sample, gain $(A)$ can be a phase angle introduced by the sample y using phase locked loop. By measuring
Table 1. Bulk resistances measured for ombinations.

istance

\begin{tabular}{|c|c|c|c|c|c|}
\hline S1. No. & $R$ & $C$ & Mode & & Standard value \\
\hline 1. & $1 \mathrm{M} \Omega$ & $200 \mathrm{pF}$ & Parallel & ${ }^{2} \mathrm{M} \Omega$ & $1 \mathrm{M} \Omega$ \\
\hline 2. & $8 \cdot 2 \mathrm{M} \Omega$ & $200 \mathrm{pF}$ & Parallel & $8 \cdot 19 \pm 10^{-2} \mathrm{M} \Omega$ & $8 \cdot 2 \mathrm{M} \Omega$ \\
\hline 3. & $4.8 \mathrm{M} \Omega$ & $50 \mathrm{pF}$ & Parallel & $4 \cdot 79 \pm 10^{-2} \mathrm{M} \Omega$ & $4.8 \mathrm{M} \Omega$ \\
\hline 4. & $10 \mathrm{~K} \Omega$ & $100 \mathrm{pF}$ & Series & $9 \cdot 81 \pm 10^{-5} \mathrm{~K} \Omega$ & $10 \mathrm{~K} \Omega$ \\
\hline
\end{tabular}


gain and phase angle, impedance of the sample is calculated which can be resolved into real and imaginary parts as

$$
\begin{aligned}
& Z^{\prime}=|Z| \cos \phi, \\
& Z^{\prime \prime}=|Z| \sin \phi,
\end{aligned}
$$

Table 2. Bulk resistances of the polymer and polycrystalline samples (pellets) measured in the present study.

\begin{tabular}{ll}
\hline Sample & Bulk resistance \\
\hline $1 \cdot 5 \% \mathrm{NaF}+\mathrm{KYF}_{4}$ & $45 \cdot 95 \pm 10^{-5} \mathrm{~K} \Omega$ \\
$2 \% \mathrm{NaF}+\mathrm{KYF}_{4}$ & $84 \cdot 7 \pm 10^{-2} \mathrm{M} \Omega$ \\
$\mathrm{PEO}+\mathrm{KYF}_{4}$ & $10 \cdot 3 \pm 10^{-2} \mathrm{M} \Omega$ \\
$\mathrm{PEO}+\mathrm{NaYF}_{4}$ & $26 \cdot 24 \pm 10^{-2} \mathrm{M} \Omega$ \\
\hline
\end{tabular}

$\mathrm{NaF}$, sodium fluoride; $\mathrm{KYF}_{4}$, potassium yttrium tetraflouride; $\mathrm{NaYF}_{4}$, sodium yttrium tetraflouride; PEO, poly ethylene oxide. where

$$
|Z|=R_{\mathrm{f}} /|A| \text {. }
$$

The real and imaginary impedances are evaluated as a function of frequency. The data collected is analysed in the form of impedance plots. From the complex impedance plots bulk resistance of the sample is obtained. Finally a.c. conductivity is calculated by using the relation,

$$
S=t / R \cdot a,
$$

where $t$ is the thickness, $a$ the area of cross-section and $R$ the bulk resistance of the sample.

\section{Calibration}

In the present investigation, the phase angle and gain are measured as functions of frequency for different series and parallel combinations of known values of resistors and capacitors in order to study the reliability of the experimental technique developed. The results (table 1) are found to be in good agreement with the standard results within experimental error.
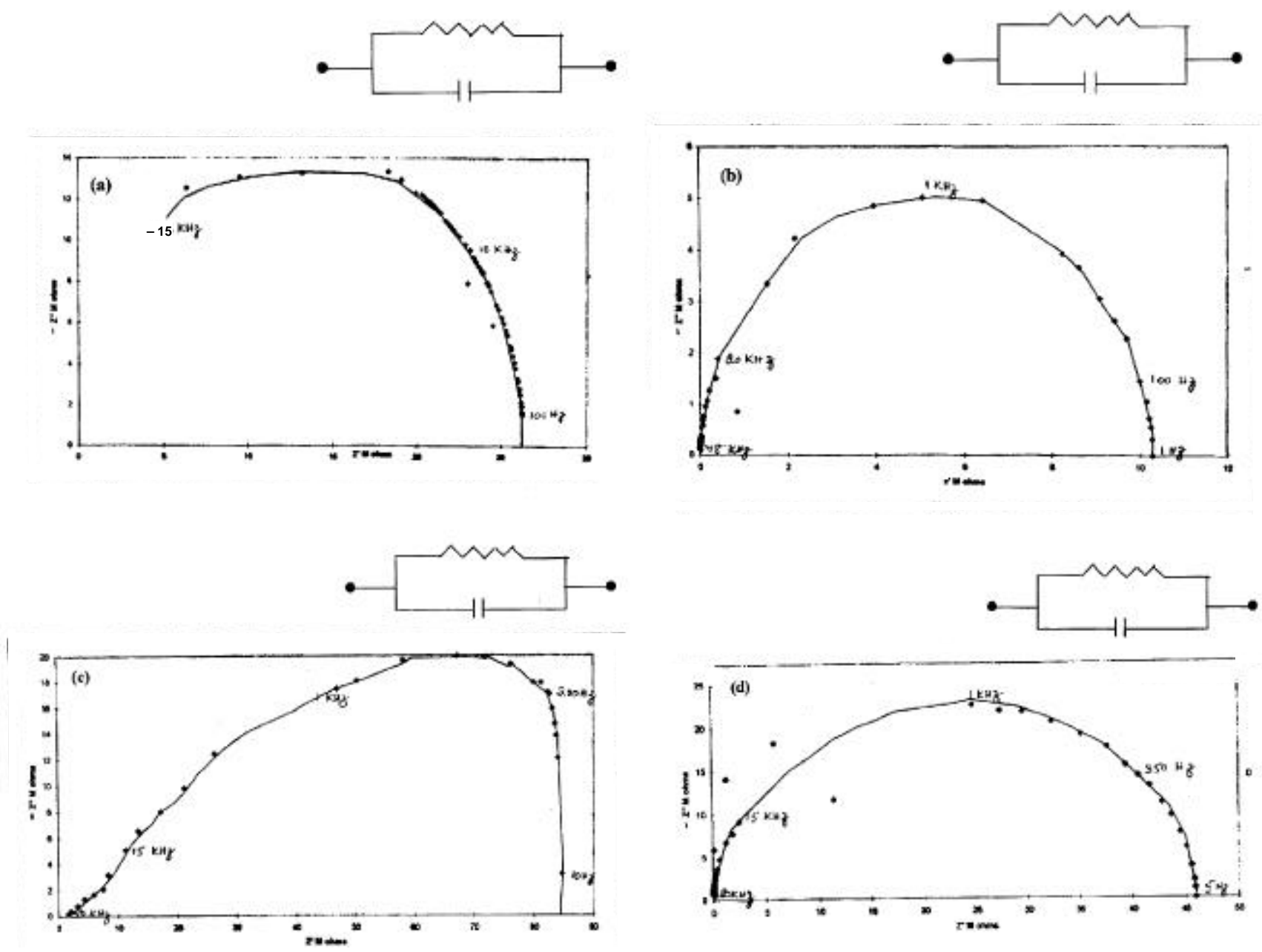

Figure 7. (a) Complex impedance plot of $\mathrm{PEO}+\mathrm{NAYF}_{4}$; (b) $\mathrm{PEO}+\mathrm{KYF}_{4}$; (c) $2 \% \mathrm{NaF}+\mathrm{KYF}_{4}$ and (d) $1 \cdot 5 \% \mathrm{NaF}+\mathrm{KYF}_{4}$. 


\section{Results}

In order to standardize the developed technique, polymer and polycrystalline samples in which bulk resistances have already been measured (Sreepathi Rao et al 1994) are chosen. Bulk resistances measured in the present study from impedance plots for the above samples are shown in table 2. The impedance plots for the samples studied, and their equivalent circuits are shown in figure 7.

\section{Conclusions}

From the results obtained in the present study, it is clear that measurements made using the technique developed for the known combinations of resistor and capacitor predict the nature of electrical equivalent circuit and their parameters. The studies made on the samples were compared with the results of Shareefuddin (1993).

Using the above developed instrument, complex impedance spectroscopy measurement studies in the frequency range of $1 \mathrm{~Hz}$ to $80 \mathrm{kHz}$ is in progress.

\section{Acknowledgements}

The authors thank Prof. A B Kulkarni, Department of Applied Electronics, Gulbarga University, Gulbarga, for his keen interest in the present work. They also thank the referee for his useful comments.

\section{References}

Balaya P and Sunandana C S 1989 Pramana - J. Phys. 33627

Bauerle J E 1969 J. Phys. Chem. Solids 302657

Boukamp B A 1984 Solid State Ionics 11339

Bruce P G and West A R 1983 J. Elec. Chem. Soc. 1303

Burr-Brown 1998 IC Data Book

Macdonald J R (ed.) 1987 Impedance spectroscopy emphasizing solid state materials and systems (New York: Wiley)

National Semiconductor Corporation 2000 Linear IC Data Book 33

Schon G 1989 Solid State Ionics 2231

Shareefuddin 1993 Transport properties of potassium yttrium fluoride and sodium yttrium fluoride materials, Ph.D. Thesis, Osmania University, Hyderabad

Sreepathi Rao S, Jaipal Reddy M, Narasimha Reddy K and Subba Rao U V 1994 Solid State Ionics 74225

Staudt U 1981 Solid State Ionics 2175 